\title{
Frontières
}

\section{La « bonne mort »}

\section{Jocelyne Saint-Arnaud}

Volume 20, numéro 1, automne 2007

La « bonne mort »

URI : https://id.erudit.org/iderudit/017940ar

DOI : https://doi.org/10.7202/017940ar

Aller au sommaire du numéro

Éditeur(s)

Université du Québec à Montréal

ISSN

1180-3479 (imprimé)

1916-0976 (numérique)

Découvrir la revue

Citer ce document

Saint-Arnaud, J. (2007). La « bonne mort ». Frontières, 20(1), 7-8.

https://doi.org/10.7202/017940ar

Ce document est protégé par la loi sur le droit d'auteur. L'utilisation des services d'Érudit (y compris la reproduction) est assujettie à sa politique d'utilisation que vous pouvez consulter en ligne.

https://apropos.erudit.org/fr/usagers/politique-dutilisation/
Cet article est diffusé et préservé par Érudit.

Érudit est un consortium interuniversitaire sans but lucratif composé de l’Université de Montréal, l'Université Laval et l'Université du Québec à Montréal. Il a pour mission la promotion et la valorisation de la recherche. https://www.erudit.org/fr/ 


\section{LA \\ «BONNE MORT »}

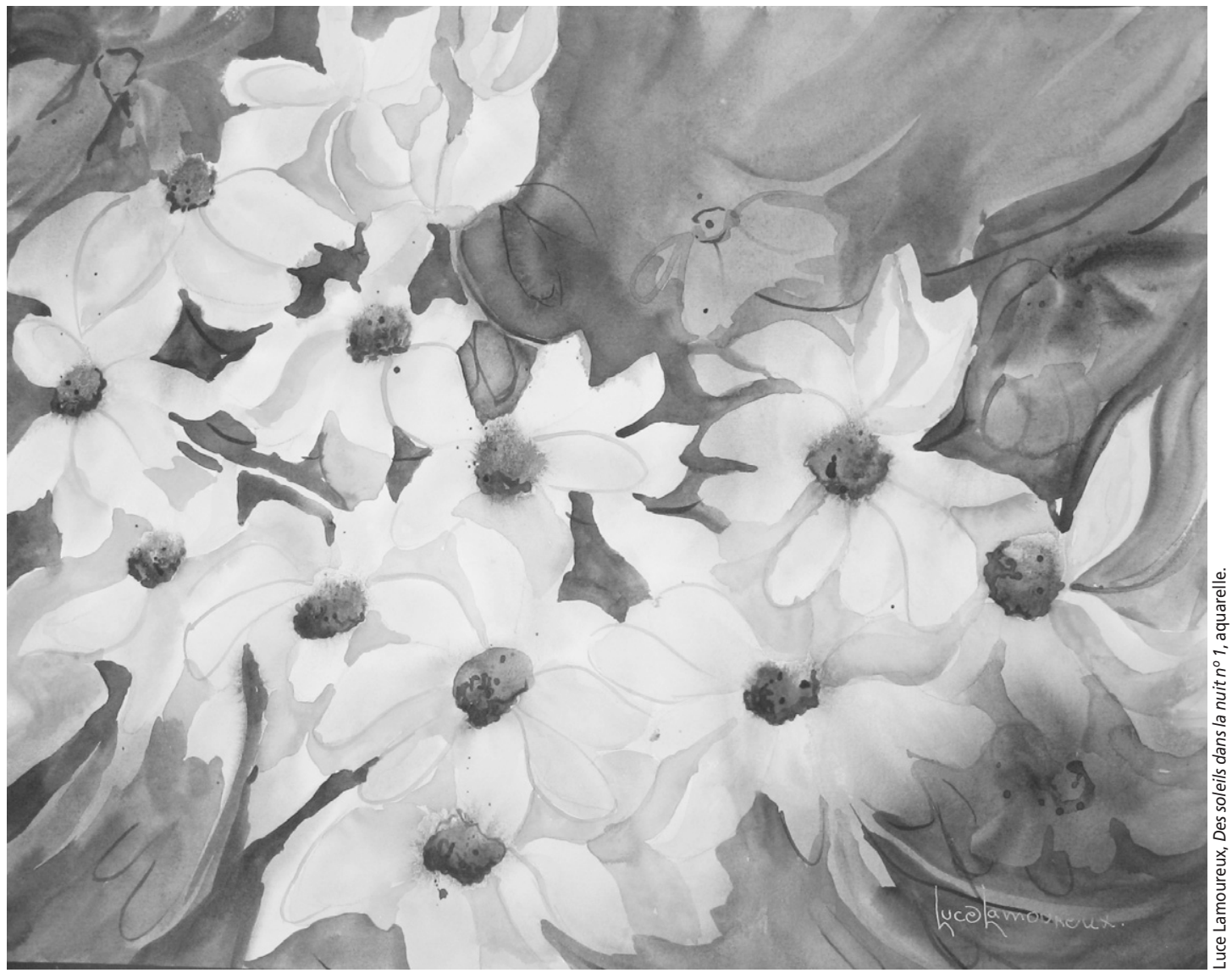

Jocelyne Saint-Arnaud, Ph. D., directrice du numéro.

La «bonne mort», certains n'y croient pas. Pourtant des études auprès de personnes en fin de vie indiquent qu'en général on veut mourir rapidement, dans son sommeil et surtout sans douleur. On répète souvent que ce n'est pas la mort en elle-même qui est l'objet de craintes, mais ce qui la précède. Parler de la mort n'est pas aisé. Plusieurs auteurs ont analysé le tabou qui entoure la fin de vie, la mort cachée, la mort qui tue et qui est tue. Dans ce numéro, des auteurs se commettent sur le sujet selon des perspectives diversifiées. Le numéro s'ouvre sur une étude des fragments d'Héraclite d'Éphèse sur la mort. Paradoxalement, celui qui fut surnommé l'Obscur nous éclaire aujourd'hui sous la plume d'Éric Volant. Le philosophe du devenir, de l'éternel retour, de l'harmonie des contraires présente la mort comme inséparable de la vie: "la vie et la mort sont une seule et même chose; de même la veille et le sommeil; la jeunesse et la vieillesse; car les premiers de ces états sont devenus seconds et les seconds à rebours devenus les premiers » (DK 88). La vision du temps chez Héraclite est circulaire, voire mythique, alors que chez Dominique Jacquemin, l'histoire s'inscrit dans la religion par le mystère de la mort et de la résurrection du Christ. Celui qui a connu, en tant qu'homme, la douleur de la mort peut nous soutenir dans la peine, la maladie et la faiblesse qui accompagnent toute mort. Autre perspective sur le temps, celui du mourir. L'expérience qu'on en a, selon la visée anthropologique de Luce Des Aulniers, est fonction de la traversée de la maladie grave, voire de toute la vie antérieure, consciemment, inconsciemment, voire préconsciemment.

La «bonne mort» serait-elle une utopie ? Pour répondre à cette question, Serge Daneault analyse les résultats d'une revue intégrative des écrits (Kehl, 2006) qui énumère les douze caractéristiques d'une bonne mort, dont le contrôle, le confort, le sentiment d'être une personne à part entière jusqu'à la fin. 
Critique face aux services de soins palliatifs offerts dans une unité européenne de soins palliatifs, où elle a fait de l'observation pendant un mois, Florence Ollivier analyse le cas des personnes qui n'entrent pas dans le modèle par étapes de Kübler-Ross. Elle présente notamment le cas de l'«im-patient», celui qui veut mourir plus vite et sans soutien psychologique. Schiffino et Bert analysent la loi belge qui dépénalise l'euthanasie et l'aide au suicide, alors que Brian Mishara examine, de manière critique, les arguments en faveur d'une légalisation de l'aide au suicide et de l'euthanasie ${ }^{1}$. Il en conclut que s'il y avait légalisation de tels actes au Canada, il serait préférable de légaliser l'aide au suicide parce qu'elle préserve une plus grande autonomie, notamment dans la possibilité de se raviser.

La section Recherche débute par une analyse des résultats d'un recherche-action portant sur les besoins d'apprentissage des bénévoles en contexte de soins palliatifs pédiatriques. Après avoir répertorié les besoins et les éléments à retenir pour créer un programme de formation pour les bénévoles, un comité formé de huit membres a implanté et évalué ce nouveau programme. Manon Champagne y définit, entre autres, les spécificités du bénévolat dans un contexte de soins palliatifs dans un milieu pédiatrique. Dans un tout autre domaine, non moins médicalement interventionniste, Sylvie Décarie et ses collègues présentent le développement d'une approche intégrée de soins curatifs et palliatifs auprès d'une clientèle atteinte d'insuffisance cardiaque sévère. Isabelle Marcoux présente les résultats d'une enquête téléphonique effectuée auprès de 991 adultes québécois et portant sur les attitudes face à l'euthanasie. On y apprend que 69,6\% des répondants jugent l'euthanasie acceptable; parmi eux, 37,6\% la jugent acceptable même en l'absence de maladie incurable et $27 \%$ en l'absence de douleur. Finalement, un article de Johan Bilsen et ses collègues, publié dans la revue Social Science and Medicine en 2007, a été traduit pour le bénéfice des lecteurs francophones. Cet article traite des changements dans les pratiques médicales liés au processus de dépénalisation de l'euthanasie en Belgique. On y apprend notamment que, depuis 2004, les médecins consultent davantage les patients, leur famille et le personnel infirmier au sujet de décisions de traitements susceptibles de raccourcir la vie.

La section Intervention s'ouvre sur un projet d'un comité d'éthique clinique, celui du CSSS de Rouyn-Noranda, qui vise à faciliter la communication des volontés relatives à l'intensité des niveaux de soins. Ses auteures, Sarah Shidler et Annie Léger, y analysent une situation éthique problématique. L'article se termine par un encouragement destiné aux patients, leur famille, et aux professionnels de la santé à discuter des décisions de soins et de traitements en fin de vie en temps opportun. Saint-Arnaud et ses collègues font état de la situation des soins palliatifs au Québec et des besoins de formation universitaire interdisciplinaire dans ce domaine. Enfin, Federico Palermiti présente un plaidoyer en faveur du respect des droits des personnes atteintes de la maladie d'Alzheimer.

La section Points de vue regroupe des témoignages et réflexions sur la vie et la fin de vie. D'abord un témoignage de Marité Villeneuve sur l'accompagnement d'une personne atteinte d'Alzheimer; il s'agit de l'expérience vécue par une dame et sa fille aidante, psychologue et écrivaine de profession. Ce récit expose la fragilité et la finitude de la personne humaine, le besoin de protection de la personne atteinte de l'Alzheimer, mais surtout son besoin d'être reconnue et aimée comme personne à part entière. Et n'est-ce pas cette crainte de la perte du sens, de la reconnaissance d'être une personne humaine à part entière, de l'emprise de la technique sur la fin de vie, qui motive les humains à désirer la mort? Dans un autre témoignage, Claire Morissette, irrémédiablement atteinte, livre ses sensations et émotions face à ses pertes, sa douleur, son quotidien de personne «condamnée à mort ", selon son expression. Des appels à la mort, comme celui de Claire Morissette aujourd'hui décédée, trouvent écho chez les tenants d'un droit à la mort dans la dignité. Dans la suite du projet de loi C-407 de la députée Francine Lalonde, visant à amender le code criminel pour permettre à une personne qui le désire d'obtenir l'aide d'un médecin ou d'une tierce personne pour «mourir dignement», Guy Lamarche présente une nouvelle association québécoise pour la défense du droit de mourir dans la dignité. Deux médecins retraités, Marcel Boulanger et Marcel Boisvert confient leur point de vue sur la question. S'ajoute en hors thème le témoignage d'une mère, Hélène Panneton, qui a donné un rein à son fils; cette expérience vécue comme un second accouchement apporte un baume sur notre "humanitude». Les textes regroupés dans cette section sont très subjectifs et c'est ce qui les distingue des articles des sections précédentes. Cependant, ils ne sont pas étrangers à la recherche dans le domaine des études sur la mort, puisque toute étude de type scientifique, notamment l'approche narrative de plus en plus utilisée en recherche dans le domaine des soins de santé, s'alimente à des récits pour comprendre les attitudes, les perceptions et les expériences, tant des malades que des soignants.

La section Regards comporte un poème de Marie-Hélène Lindor, médecin en soins palliatifs ainsi que des extraits d'un spectacle-lecture de Marie-Louise Leblanc des productions «et Jules à mes côtés...", à partir de textes tirés des livres d'Édith Fournier intitulés J'ai commencé mon éternité et La mère d'Édith parus en $2007^{2}$ aux Éditions de l'Homme. Les expériences d'accompagnement relatés dans ces livres ont inspiré à leur auteure, Édith Fournier, des réflexions qui touchent au prendre soin, au sens de la vie et de la mort, à l'attachement. Le numéro est illustré par l'aquarelliste québécoise Luce Lamoureux qui a réalisé plusieurs expositions solos depuis 1994. Ses bouquets de fleurs apportent au numéro une dimension esthétique essentielle à la réalité humaine, quel que soit le sens qu'on y accorde.

\section{Notes}

1. Bien qu'il existe un débat sur la signification exacte des termes dépénalisation et légalisation en droit, on accepte généralement une gradation dans le passage de l'interdiction totale à l'autorisation totale et sans conditions d'une pratique. Selon $\mathrm{M}^{\mathrm{e}}$ Gary Mullins, consulté à ce sujet, cette gradation part de la pénalisation, passe par la dépénalisation pour arriver à la légalisation. Selon lui, le passage de la pénalisation à la dépénalisation fait en sorte qu'un acte dépénalisé cesse d'être sanctionné au plan pénal à certaines conditions, tout en demeurant potentiellement objet de sanctions administratives, civiles ou disciplinaires.

2. Dans le cas de La mère d'Édith, il s'agit d'une réédition, la première édition remonte à 1983 chez Libre Expression. 